\title{
Validation of capture yield calculations in the Resolved Resonance Energy Range with CONRAD code
}

\author{
Olivier Litaize ${ }^{1}$, a Pascal Archier ${ }^{1}$, Bjorn Becker ${ }^{2}$, Peter Schillebeeckx ${ }^{2}$, and Stefan Kopecky ${ }^{2}$ \\ ${ }^{1}$ CEA, DEN, Cadarache, F-13108 Saint Paul Lez Durance, France \\ ${ }^{2}$ EC-JRC-IRMM, Retieseweg 111, B-2440 Geel, Belgium
}

\begin{abstract}
This paper deals with the validation of the multiple scattering corrections developed in the CONRAD code for the capture yield calculations in the Resolved Resonance energy Range (RRR). In order to calculate the capture yields, analytic and stochastic calculation schemes implemented in CONRAD are described and compared with the analysis code SAMMY/SAMSMC. The results are in excellent agreement for a variety of samples. We concentrate the discussion here on ${ }^{238} \mathrm{U},{ }^{197} \mathrm{Au}$ and ${ }^{55} \mathrm{Mn}$.
\end{abstract}

\section{Introduction}

CONRAD [1] is dedicated to nuclear reaction cross section evaluation and related variance-covariance matrices from thermal up to the fast energy range. The aim of this note is to validate the capture yield calculations in the Resolved Resonance energy Range (RRR).

The cross section calculations performed with CONRAD are compared with the reference code NJOY [2]. Transmissions and capture yields are compared with the Resonance Shape Analysis codes REFIT [3] and SAMMY/SAMSMC [4]. The nuclear reaction models implemented in the code for the RRR are the Reich-Moore and the Multilevel Breit-Wigner approximations of the $R$-Matrix theory. The details of the validation of cross section and transmission calculations can be found in Refs. [5]-[7]. In general, the calculation with CONRAD are in perfect agreement with NJOY using the Multilevel Breit-Wigner or the Reich-Moore nuclear reaction model. As a transmission is a trivial functional of the total cross section, its calculation is also in excellent agreement and has been checked against REFIT code in Ref. [7]. In capture measurements, due to neutron energy loss in the sample, an additional experimental correction is made: the multiple scattering corrections.

After a short description of the problematics in section 2, comparisons for ${ }^{238} \mathrm{U},{ }^{197} \mathrm{Au}$ and ${ }^{55} \mathrm{Mn}$ samples are presented in sections 3, 4 and 5, respectively.

\section{Calculation route for capture yields}

We concentrate on time-of-flight capture measurements (see for instance Ref. [9] for details of such a measurement performed at GELINA in Geel, Belgium). If we leave aside the experimental resolution (discussed in these proceedings in Ref. [8]) due to detector, moderator, ..., which can be neglected in

ae-mail: olivier.litaize@cea.fr 
various configurations, we must be able to calculate as precisely as possible the capture yield. This is the main goal of this paper.

The capture yield of a neutron at energy $E$ by a nucleus can be decomposed in three parts:

$$
Y(E)=Y_{0}(E)+Y_{1}(E)+Y_{n}(E)
$$

where $Y_{0}(E)$ stands for the primary capture yield (neutron is directly captured without being scattered), $Y_{1}(E)$ stands for the single scattering correction (neutron is captured after one single scattering) and $Y_{n}(E)$ stands for the double-plus scattering correction (neutron is captured after at least two scatterings). The mathematical expression for the primary capture yield is the simplest one. It is the product of the probability for the neutron to travel up to a distance $z$ in the sample without interaction and the probability of this neutron to be captured between $z$ and $z+d z$ :

$$
Y_{0}(E)=\int_{z=0}^{z=L} e^{-\Sigma_{t} z} \Sigma_{\gamma} d z=\left(1-e^{-n \sigma_{t}}\right) \frac{\sigma_{\gamma}}{\sigma_{t}}
$$

where $\sigma$ are the microsopic cross sections in [b], $\Sigma$ are the macrosopic cross sections in $\left[\mathrm{cm}^{-1}\right], L$ is the sample thickness in $[\mathrm{cm}]$ and $n$ stands for the sample thickness in [at/b]. The nucleus concentration $N$ in $[\mathrm{at} / \mathrm{b} / \mathrm{cm}]$ is given by $N=n / L$. The expression of the single scattering correction $Y_{1}$ is a bit more complicated and corresponds to a six-fold embedded integration over sample and beam geometries. Details can be found in Ref. [10]. Due to its complexity, the computation of the $Y_{n}$ component requires at least two approximations: a uniform spatial distribution and an isotropic angular distribution of the neutrons in the sample after two scatterings. This double-plus scattering correction is valid for thin samples where $Y_{0}$ and $Y_{1}$ are the predominant corrections (see also [10] for details).

At the time being, only analytic $Y_{0}$ and $Y_{1}^{\infty}$ components are implemented in the code. $Y_{1}^{\infty}$ corresponds to infinite samples, or samples with radius larger than beam radius $R_{s}>>R_{n}$ in regard to the neutron mean free path.

A Monte Carlo simulation (MC) of the neutron transport in the sample is also available in CONRAD for all multiple scattering corrections. A non-analog simulation with implicit capture and russian roulette was implemented in the code. This means that at each interaction event, the probability of neutron capture is taken into account by reducing the neutron weight according to the ratio of capture and total cross section. The scattered neutron is tracked down to a given statistical weight limit depending on the roulette threshod. This limit is implemented in order to avoid too time consuming long histories.

The simulation can be performed in time or in energy using different kind of tallies. We have to keep in mind that the capture yield given by Eq. (1) is the proportion of neutrons captured at energy $E$ even if they are captured after one or more scatterings (and then having an energy $E^{\prime}$ ). Consequently Monte Carlo simulation will be comparable with analytic formulae only if the tally corresponds to the initial energy of the neutron whatever its actual energy ('tof-fixed').

This means that time is fixed at the entrance in the sample and because the distance used to obtain an energy spectrum is fixed to the flight length, this corresponds to a simple simulation in energy tallying the score at the incoming neutron energy: this is fully consistent with the analytic formulae. On the other hand, if we want to simulate a time-of-flight experiment, we have to account for the actual neutron traveling time in the sample and to use a fixed distance (the flight length between neutron source and the entrance surface of the sample) to transform the time spectrum in an energy spectrum ('tof-varying'). These two kind of simulations are not the same but the difference is so tight that it can be detected only with a thick sample. This has to be kept in mind when we will compare analytic calculations, Monte Carlo simulations and time-of-flight measurements. 


\section{$3{ }^{238} \mathrm{U}$ primary capture yield and single scattering correction}

The primary capture yield and single scattering corrections for a $0.5 \mathrm{~cm}$ thick ${ }^{238} \mathrm{U}$ cylindrical sample $\left(n \sim 3.10^{-3} \mathrm{at} / \mathrm{b}\right.$ ) are calculated with analytic and stochastic schemes. The $J^{\pi}=1 / 2^{+}$s-wave neutron resonance at $36.7 \mathrm{eV}$ is shown here. The radius of the sample is large enough compared to the beam radius that the $Y_{1}$ contribution can be calculated within the infinite approximation.

Figure 1 shows a comparison of $Y_{0}$ calculated by applying Eq. (2) with NJOY based cross sections, by using the CONRAD 'analytic scheme' and by performing MC simulations either in time or in energy (see section 2). We can observe the slight discrepancy between the MC simulations for this kind of unusually thick sample. Here we concentrate on comparison between analytic and stochastic calculations: the agreement is excellent.

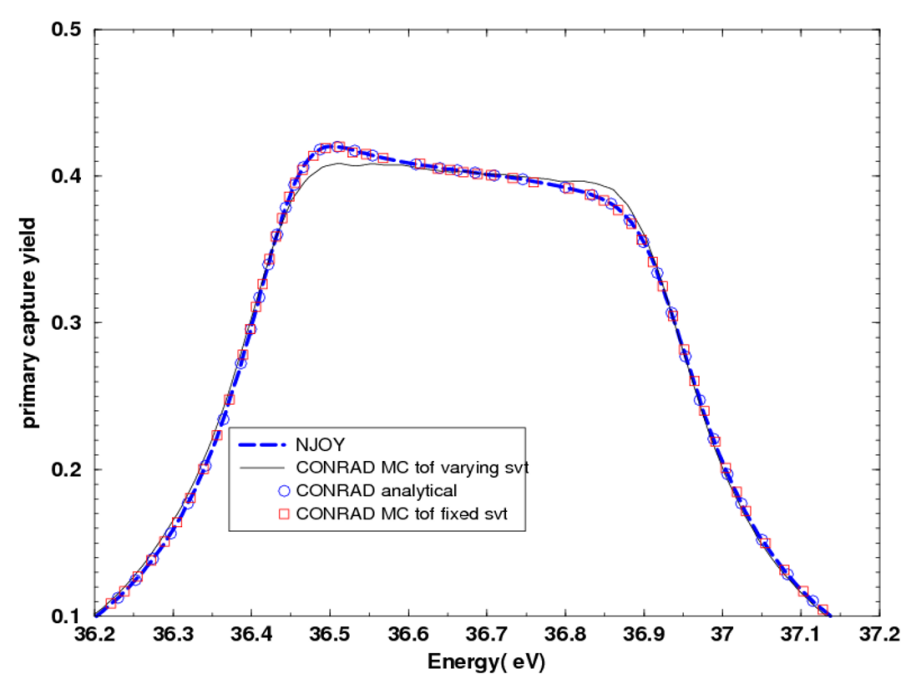

Figure 1. Calculation of the primary Capture Yield of ${ }^{238} \mathrm{U}$ with various schemes.

Figure 2 shows the single scattering correction obtained without considering edge effects (infinite slab approximation) in the analytic calculation. The result of the MC simulation is in perfect agreement with that of the analytic scheme demonstrating the validity of the infinite approximation for this specific case. In addition we can disentangle the contributions of the captures after a backward scattering and those after a forward scattering. The different shapes are essentially due to the kinematics and the relative importance of the microscopic cross sections in the corresponding energy range.

\section{$4{ }^{197}$ Au capture yield}

The different components of the capture yield are calculated with CONRAD in the case of a cylindrical gold sample $\left(N \sim 6.10^{-4} \mathrm{at} / \mathrm{b} / \mathrm{cm}\right)$ at $4.9 \mathrm{eV}\left(J^{\pi}=2^{+}\right)$. The results are compared with those from SAMMY. Again the sample is sufficiently thick (few centimeters) and the radial dimension sufficiently large compared to the beam radius to support the validity of an 'infinite slab approximation'. The primary capture yield $Y_{0}$ and single scattering correction $Y_{1}$ are calculated with the analytic scheme with both CONRAD and SAMMY codes. The double-plus scattering correction and the total capture yield are calculated in CONRAD with the Monte Carlo scheme. 


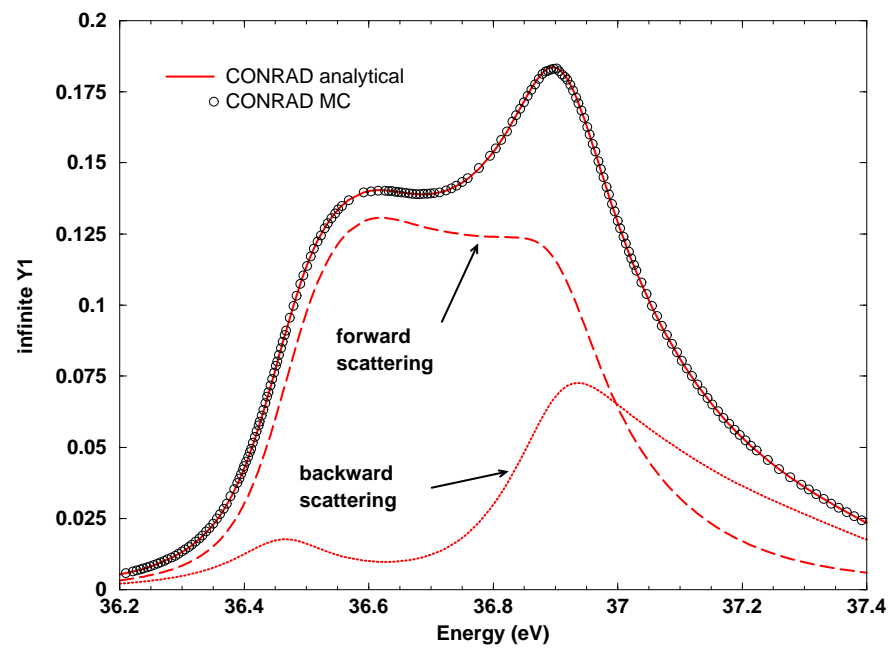

Figure 2. Single scattering correction for the capture yield of ${ }^{238} \mathrm{U}$ within the infinite sample approximation.

The results agree very well except for the double-plus scattering correction which is a well known crude approximation in the analytic schemes. Because this component is negligible in this kind of sample, there is only a little difference in the total capture yield. Figure 3 shows the comparison of the different computations.

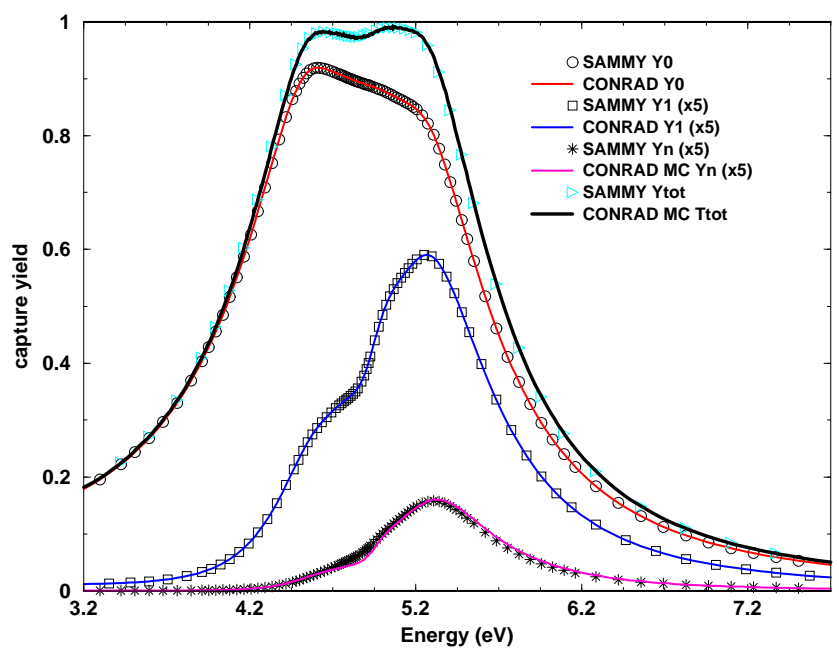

Figure 3. Multiple scattering corrections for the capture yield of ${ }^{197} \mathrm{Au}$ within the infinite slab approximation. 


\section{$5{ }^{55} \mathrm{Mn}$ capture yield}

The $J^{\pi}=2^{-}$s-wave resonance of ${ }^{55} \mathrm{Mn}$ at $337.3 \mathrm{eV}$ is studied in this section. The sample is a 3 $\mathrm{mm}$ thick cylinder $\left(N \sim 6.10^{-2} \mathrm{at} / \mathrm{b} / \mathrm{cm}\right)$. This sample and energy range is particularly suited to test the multiple scattering correction due to a strong scattering component of the resonance. Figure 4 shows the various components of the capture yield calculated with the MC schemes of CONRAD and SAMMY/SAMSMC (the only parameters of this single resonance are used here for a proper benchmark description). The relative magnitude of $Y_{n}$ is around 6 times higher than $Y_{0}$ (the neutron width $\Gamma_{n}(\sim 22 \mathrm{eV})$ is much stronger than the radiative capture width $\left.\Gamma_{\gamma}(310 \mathrm{meV})\right)$, this is the reason why analytic schemes fail to reproduce the good shape of this yield [11]. Figure 5 shows a comparison of the capture yield calculated with the MC scheme of CONRAD using the whole set of resonances from JEFF-3.1.1 library and the preliminary results of the measurements from IRMM [12]. The shape is very well reproduced with the MC simulation, except in the wings. However,we have to keep in mind that there is no experimental resolution broadening in the calculation.

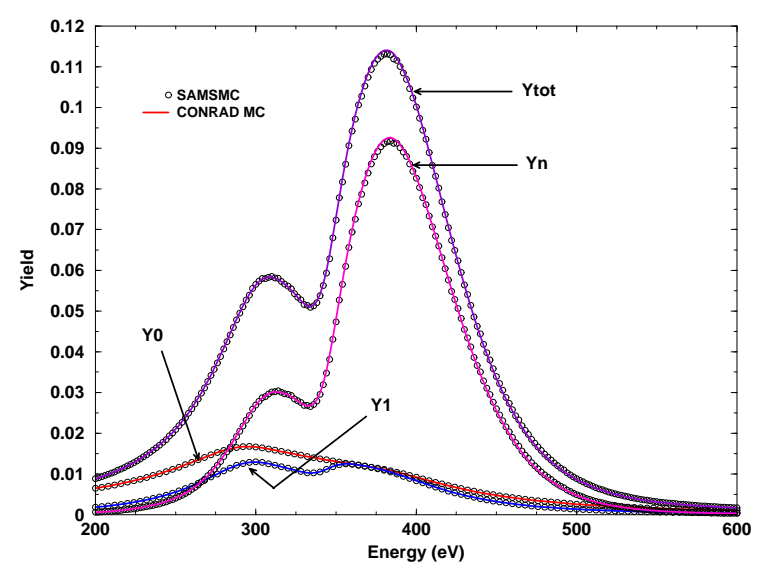

Figure 4. Multiple scattering corrections for the capture yield of ${ }^{55} \mathrm{Mn}$.

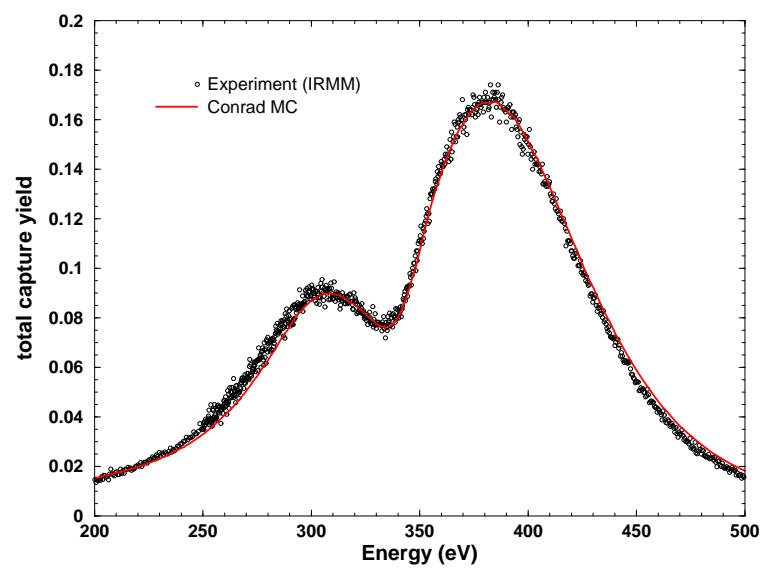

Figure 5. Total capture yield of ${ }^{55} \mathrm{Mn}$. Comparison with experiment (IRMM). 
EPJ Web of Conferences

\section{Conclusion}

Capture yield computations have been performed with CONRAD using analytic and Monte Carlo methods and have been compared with analysis codes like REFIT, SAMMY and SAMSMC. The primary capture yield calculated with both analytic and Monte Carlo schemes in CONRAD are in perfect agreement with those of SAMMY/SAMSMC codes. The single scattering correction within the infinite slab approximation of the analytic scheme is also in excellent agreement with the reults of the SAMMY code. The other multiple scattering corrections available through Monte Carlo simulations in CONRAD are in good agreement with those of SAMMY/SAMSMC. The weak point of analytic formulae are the hypothesis of uniform distributions for $Y_{n}$ and the asymptotic scattering kernel (target at rest) used even for $Y_{1}$. The future works to be done in CONRAD concern among others the analytic $Y_{1}$ (with edge effects) and $Y_{n}$ and the use of a refined scattering kernel model such as the Doppler Broadening Rejection Correction (DBRC) model [13] in order to treat the resonant scattering in the Monte Carlo scheme.

\section{Acknowledgements}

One of the authors (OL) whishes to thank M.C. Moxon, R. Dagan, G. Noguere and C. De Saint Jean for fruitfull discussions.

\section{References}

[1] C. De Saint Jean, B. Habert, O. Litaize, G. Noguère and C. Suteau, Status of Conrad, a nuclear reaction analysis code, in Proc. of the Int. Conf. on Nucl. Data for Science and Technology ND2007, Nice, France, p251 (2007)

[2] R.E. MacFarlane, update web site. http://t2.lanl.gov/codes/njoy99/index.html, 21 February 2011

[3] M.C. Moxon, Harwell Laboratory Technical Report AEA-IN Tec=0470, 1991

[4] N.M. Larson, Oak Ridge National Laboratory Report ORNL/TM-9179/R7, 2005

[5] O. Litaize, C. De Saint Jean, G. Noguere, B. Habert and C. Suteau, Technical Note DEN/CAD/DER/SPRC/LEPH NT-08/217, 2008.

[6] C. De Saint Jean et al., http://www.oecd-nea.org/dbdata/process/workshop-2008, Workshop on processing, Issy-Les-Moulineaux, France, 2008.

[7] O. Litaize, C. De Saint Jean and G. Noguere, Technical Note DEN/CAD/DER/SPRC/LEPH NT09/226, 2009.

[8] P. Archier, G. Noguere, O. Litaize, C. De Saint Jean, P. Schillebeeckx, S. Kopecky and K. Volev, in Proc. of this Workshop, 2012.

[9] P. Schillebeeckx, A. Borella, S. Kopecky, C. Mampoudis, C. Massimi, M. Moxon, J. of the Korean Phys. Soc., 59, No. 2, pp. 1563-1568 (2011)

[10] N. Larson and K. Volev, in Proc. of the Int. Conf. on Nuclear Data for Science and Technology ND2002, Seoul, Korea (2002)

[11] P. Schillebeeckx, Status WG36, WPEC, NEA Headquarters, 12-13 May 2011.

[12] P. Schillebeeckx, private communication, 2012.

[13] B. Becker, R. Dagan, G. Lohnert, Ann. Nucl. Ener. 36, pp. 470-474 (2009). 